\title{
Activation of insulin receptors and IGF-1 receptors in COLO-205 colon cancer xenografts by insulin and insulin analogue X10 does not enhance growth under normo- or hypoglycaemic conditions
}

\author{
Henning Hvid ${ }^{1}$ (D) $\cdot$ Mikkel S. Jørgensen $^{1} \cdot$ Niels Blume ${ }^{1} \cdot$ Rita Slaaby $^{1} \cdot$ Anne Lützen $^{1} \cdot$ Bo F. Hansen ${ }^{1}$
}

Received: 2 May 2018 / Accepted: 4 June 2018 / Published online: 12 July 2018

(C) Springer-Verlag GmbH Germany, part of Springer Nature 2018

\begin{abstract}
Aims/hypothesis Recent studies with normal rats and mouse allograft models have reported that insulin and insulin analogues do not activate the IGF-1 receptor in vivo, and that this characteristic therefore cannot be responsible for the increased incidence of mammary tumours observed for the insulin analogue X10 in chronic toxicity studies with Sprague Dawley rats. This is in clear contrast to reports of insulin and insulin analogues in vitro. Clarification of this is important for understanding the mechanisms behind possible growthpromoting effects of insulin analogues, and will have implications for the development of novel insulin analogues.

Methods We established a xenograft model in BALB/c nude mice with the human colon cancer cell line COLO-205, which expresses human insulin and IGF-1 receptors, and explored the acute and chronic effects of treatment with suprapharmacological doses of human insulin, insulin analogue X10 and human IGF-1. With a novel antibody, acute IGF-1 receptor activation was also examined in various tissues from normal rats treated with human insulin, insulin analogue X10 or human IGF-1. Finally, the effects of pharmacologically relevant doses of human insulin and insulin analogue X10 on receptor activation and growth of COLO-205 xenograft were explored in BALB/c nude mice with alloxan-induced hyperglycaemia.

Results In normal rats and in BALB/c nude mice bearing a COLO-205 cell xenograft, treatment with supra-pharmacological doses of human insulin, insulin analogue X10 or human IGF-1 resulted in activation of insulin receptors as well as IGF-1 receptors. Treatment of diabetic nude mice with pharmacologically relevant doses of human insulin or insulin analogue X10, which decreased blood glucose from hyperglycaemic levels to the normoglycaemic range, did not increase IGF-1 receptor activation. Furthermore, repeated treatment with supra-pharmacological as well as pharmacological doses of human insulin or insulin analogue X10 did not influence the growth of COLO-205 xenografts.

Conclusions/interpretation This study demonstrates that activation of IGF-1 receptors in cancer cells by insulin and insulin analogues cannot be considered as a purely in vitro phenomenon. It does occur in vivo in animal models, although only after treatment with supra-pharmacological doses. Furthermore, treatment with insulin or insulin analogue X10 did not influence the growth of COLO-205 xenografts under normo- or hypoglycaemic conditions. Further studies are needed before a conclusion can be reached on whether IGF-1 receptor activation by insulin analogues correlates with increased growth in vivo.
\end{abstract}

Keywords IGF-1 $\cdot$ IGF-1 receptor activation $\cdot$ Insulin $\cdot$ Insulin analogues $\cdot$ Insulin receptor activation

\section{Abbreviation \\ IGF-1R IGF-1 receptor}

Electronic supplementary material The online version of this article (https://doi.org/10.1007/s00125-018-4684-1) contains peer-reviewed but unedited supplementary material, which is available to authorised users.

Henning Hvid

hhvd@novonordisk.com

1 Insulin Research, Novo Nordisk A/S, Novo Nordisk Park, DK-2760 Maaloev, Denmark

\section{Introduction}

Insulin and insulin analogues can stimulate growth of certain cells in vitro, and a potential concern has been that therapeutic use of insulin and insulin analogues may stimulate growth of neoplastic cells. This speculation was originally supported by findings with insulin analogue X10 (X10). Clinical development of this analogue was discontinued because suprapharmacological doses increased the incidence of mammary tumours in female rats [1]. Recently, therapeutic use of the insulin analogue glargine was speculated to be associated with 


\section{Research in context}

\section{What is already known about this subject?}

- Insulin analogue X10 increases the incidence of mammary tumours in female rats and this correlates with increased IGF-1 receptor binding in vitro

- Recent studies with rats and mouse allograft models report that insulin and insulin analogues are not able to activate the IGF-1 receptor in vivo, and that this characteristic therefore cannot be responsible for the increased mammary tumour incidence observed with $\times 10$

\section{What is the key question?}

- Can insulin and insulin analogues activate the IGF-1 receptor in vivo and how does this correlate with growth of a COLO-205 colon cancer xenograft model?

\section{What are the new findings?}

- Insulin and insulin analogue X10 can activate the IGF-1 receptor in vivo

- Despite activation of insulin receptors and IGF-1 receptors, insulin and X10 do not enhance growth of COLO-205 xenografts

\section{How might this impact on clinical practice in the foreseeable future?}

- Increased binding and activation of the IGF-1 receptor cannot be considered an irrelevant in vitro phenomenon and must be characterised during pre-clinical development of novel insulin analogues for therapeutic use

an increased risk of developing certain forms of cancer [2, 3]; the Outcome Reduction With Initial Glargine Intervention (ORIGIN) trial demonstrated this is not the case [4]. Overall, the human relevance of this potential concern is unknown. While it is documented that certain forms of cancer occur with increased incidence in type 2 diabetes, which correlate with endogenous hyperinsulinaemia [5-7], there are no data to support a link between therapeutic use of any type of marketed insulin preparation or insulin analogue and increased risk of cancer. Nevertheless, as insulin is a growth factor, mitogenic potential should be characterised during pre-clinical development of novel analogues and compared with X10, which is considered a 'super-mitogenic' insulin analogue. X10 displays increased binding affinity to the insulin receptor and IGF-1 receptor (IGF-1R) and decreased dissociation rate from the insulin receptor [8-10]; each of these characteristics correlates with an increased mitogenic potency in vitro $[9,11]$. However, recent studies in rodent models reported that insulin and insulin analogues are not able to activate the IGF-1R in vivo [12-15]. This is in clear contrast to in vitro observations, and clarification of this is important for understanding possible growth-stimulatory effects of insulin and insulin analogues. Furthermore, if increased IGF-1R activation can be regarded as an irrelevant characteristic, it will have implications for the development of novel insulin analogues.

The aims of the present study were to examine if treatment with native human insulin or X10 results in activation of the insulin receptor and IGF-1R in a human cancer cell line in vivo, and whether repeated treatment with human insulin or
$\mathrm{X} 10$ influences growth of a human cancer xenograft. This was explored in the COLO-205 colon cancer xenograft model. Colon cancer occurs with increased frequency in type 2 diabetes, and this is the first study where activation of insulin receptor and IGF-1R and growth of a human colon cancer xenograft after treatment with human insulin or X10 have been studied in vivo.

\section{Methods}

COLO-205 cells The mitogenic potencies of human insulin, X10 and IGF-1 were assessed in COLO-205 cells (ATCC, Manassas, VA, USA) as described previously [16]. COLO205 cells for injection in mice were trypsinised, washed once in PBS, re-suspended in PBS at a concentration of $10.0 \times 10^{6}$ cells/ml and kept on wet ice until injection.

Animal experiments All experimental procedures involving animals were performed under a licence granted by the Danish authority the Animal Experiment Inspectorate and followed the Guide for the Care and Use of Laboratory Animals [17]. Homozygous male BALB/c nude mice (CAnN.Cg-Foxn1 nu/Crl) were purchased from Charles River (Charles River Laboratories Germany, Sulzfeld, Germany) at age 7 weeks. Mice were housed, five mice per cage, in individually ventilated Techniplast GR 900 Seal Safe Plus cages (floor area: $904 \mathrm{~cm}^{2}$ ). Outbred male Sprague Dawley rats (Tac:SD) were purchased from Taconic 
Table 1 Animal experiments included in the study

\begin{tabular}{|c|c|c|}
\hline Experiment & Aim & Described in \\
\hline A & Explore time course for IGF-1R activation & Fig. 2 \\
\hline B & $\begin{array}{l}\text { Explore acute activation of IGF-1R after treatment with human insulin, } \\
\text { X10 and IGF-1 }\end{array}$ & Fig. 3 \\
\hline $\mathrm{C}$ & $\begin{array}{l}\text { Explore acute activation of IGF-1R in rat tissues after treatment with } \\
\text { human IGF-1 }\end{array}$ & Fig. 4 \\
\hline $\mathrm{D}$ & $\begin{array}{l}\text { Explore acute activation of IGF-1R in rat tissues after treatment with } \\
\text { human insulin and X10 }\end{array}$ & Fig. 4 \\
\hline $\mathrm{E}$ & $\begin{array}{l}\text { Explore growth of COLO-205 xenografts in mice treated repeatedly with } \\
\text { supra-pharmacological doses of human insulin or X10 }\end{array}$ & $\begin{array}{l}\text { Figs } 5,6,7 \text { and } \\
\quad \text { Tables } 3,4,5\end{array}$ \\
\hline $\mathrm{F}$ & $\begin{array}{l}\text { Explore growth of COLO- } 205 \text { xenografts in diabetic mice treated } \\
\text { repeatedly with human insulin or X10 via osmotic minipumps }\end{array}$ & Fig. 8 \\
\hline
\end{tabular}

(Taconic Biosciences, Ejby, Denmark) at age 6 weeks and housed with five rats per cage in type VI macrolone cages (floor area: $1800 \mathrm{~cm}^{2}$ ). Mice and rats had unrestricted access to a complete pelleted rodent diet (Altromin 1324, Brogården, Hørsholm, Denmark) and non-acidified non-chlorinated tap water. In the animal rooms the temperature was $18-24^{\circ} \mathrm{C}$, with relative humidity at $30-70 \%$, air change $8-15$ times $/ \mathrm{h}$ and the light/dark cycle was 12/12 h, changing at 06:00 hours and 18:00 hours.

Six animal experiments were included in this study (A-F; Table 1). Procedures for randomisation, blinding and sample size determination are described in the electronic supplementary material (ESM). All mice in experiments A, B, E and F were inoculated with $1.0 \times 10^{6} \mathrm{COLO}-205$ cells s.c. in the left flank on day 0 .

Experiment $\mathrm{A}$ included a total of 36 mice, divided into five groups of seven or eight mice. On day 21 after the injection of COLO-205 cells, the mice were treated s.c. with either vehicle or human IGF-1 (Increlex, Ipsen, Kista, Sweden), $600 \mathrm{nmol} /$ $\mathrm{kg}$. Mice were euthanised at either 5 (vehicle and IGF-1 treated), 15, 60 or $240 \mathrm{~min}$ after treatment (only IGF-1 treated). Blood samples were collected for assessment of blood glucose and plasma levels of human IGF-1, and COLO-205 xenografts were collected and snap-frozen in liquid nitrogen for analysis of IGF-1R activation.

Experiment B included of a total of 28 mice, divided into four groups of seven mice on day 21 after injection of COLO205 cells and treated with either vehicle, human insulin, X10 (Novo Nordisk, Bagsværd, Denmark) or human IGF-1 (Increlex, Ipsen), $600 \mathrm{nmol} / \mathrm{kg}$, by s.c. injection and euthanised after $15 \mathrm{~min}$. Blood samples were collected for assessment of blood glucose and plasma exposure, and samples of COLO-205 xenografts, colon, liver and the gastrocnemius muscle were collected for assessment of IGF-1R activation.

In experiment $\mathrm{C}$, a total of 20 rats, divided in four groups of five, were treated with either vehicle or human IGF-1 (Increlex, Ipsen) at doses of 6,60 or $600 \mathrm{nmol} / \mathrm{kg}$ by s.c. injection. The rats were euthanised $15 \mathrm{~min}$ after treatment, and samples of colon, anterior tibial and soleus muscle were collected and snap-frozen in liquid nitrogen for later assessment of IGF-1R activation.

Experiment D comprised 52 rats in total, divided into one group of seven and nine groups of five rats. They were treated with either vehicle, human insulin or X10 (Novo Nordisk) in doses of 6,60 or $600 \mathrm{nmol} / \mathrm{kg}$ or human IGF-1 $600 \mathrm{nmol} / \mathrm{kg}$ (Increlex, Ipsen) by s.c. injection, and $15 \mathrm{~min}$ later the rats were euthanised and samples of liver and colon were collected and snap-frozen in liquid nitrogen for subsequent assessment of IGF-1R and insulin receptor activation.

Experiment E comprised 181 mice in total, divided in three groups of 60, 60 and 61 mice, respectively. The design and analysis of experiment $\mathrm{E}$ is explained in detail in the ESM Methods and ESM Tables 1-3. The mice were treated with vehicle, human insulin or X10 (Novo Nordisk) $600 \mathrm{nmol} / \mathrm{kg}$, once daily for 18 days, starting on the day on which the COLO-205 cells were injected. Xenograft growth was monitored by measuring the length and width of each xenograft every second or third day during the experiment and then calculating the xenograft volume according to the formula: volume $=$ length $\times$ width $^{2} \times 0.52$. On day 2 , the effect on blood glucose $0-6 \mathrm{~h}$ after treatment was assessed in 15 vehicle-treated, 30 human-insulin-treated and 30 X10-treated

Table 2 Commercially available ELISAs for phospho-insulin receptor, phospho-IGF-1R and phospho-Akt

\begin{tabular}{|c|c|}
\hline Endpoint & ELISA \\
\hline $\begin{array}{l}\text { Phospho-insulin receptor } \\
\text { (Tyr1162/1163) }\end{array}$ & $\begin{array}{l}\text { IR (pY1162/pY1163) Phospho-ELISA Kit, } \\
\text { Human, catalogue number KHR9131, } \\
\text { Life Technologies, Carlsbad, CA, USA }\end{array}$ \\
\hline $\begin{array}{l}\text { Phospho-IGF-1R } \\
\text { (Tyr1131) }\end{array}$ & $\begin{array}{l}\text { PathScan Phospho-IGF-I Receptor } \beta \\
\text { (Tyr1131) Sandwich ELISA Kit, } \\
\text { catalogue number 7302, Cell Signaling } \\
\text { Technologies, Danvers, MA, USA }\end{array}$ \\
\hline Phospho-Akt (Ser473) & $\begin{array}{l}\text { AKT (pS473) Phospho-ELISA Kit, Human, } \\
\text { catalogue number KHO0111, Life } \\
\text { Technologies, Carlsbad, CA, USA }\end{array}$ \\
\hline
\end{tabular}




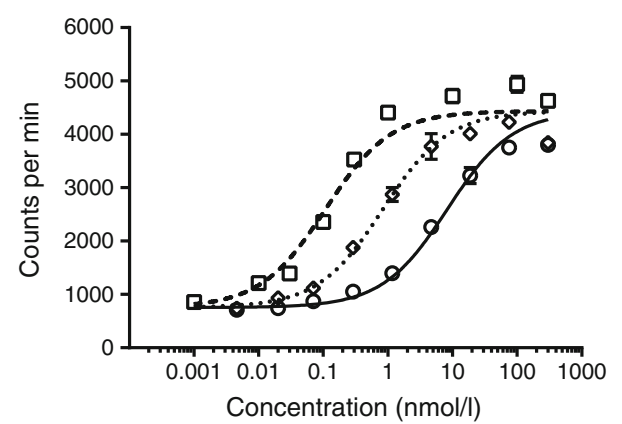

Fig. 1 Mitogenic potency in vitro of IGF-1 (squares), X10 (diamonds) and human insulin (circles) in COLO-205 cells. A representative experiment with ${ }^{3} \mathrm{H}$-thymidine incorporation in COLO-205 cells stimulated with test compounds is shown. The $x$-axis is logarithmic $\left(\log _{10}\right)$ and shows the actual concentrations of test compounds. Based on three independent experiments, the mean mitogenic potencies, including 95\% CIs, of X10 and IGF-1 relative to human insulin (i.e. fold difference), were calculated as $6.4(4.7,8.7)$ and $23.6(20.3,27.5)$, respectively. Symbols indicate mean \pm SEM based on three replicates

mice. At day 18 , mice were euthanised 5 or 15 min after the last treatment, COLO-205 xenografts were collected, weighed and snap-frozen in liquid nitrogen for later assessment of insulin receptor, IGF-1R and Akt activation and epididymal fat pads were weighed.

Experiment $\mathrm{F}$ comprised 17 normal non-diabetic mice and four groups of 13 diabetic mice each. Hyperglycaemia was induced with alloxan (Sigma-Aldrich, Brøndby, Denmark), $90 \mathrm{mg} / \mathrm{kg}$, administered i.v. Three days later (day 0), Alzet osmotic minipumps (Durect Corporation, Cupertino, CA, USA) filled with human insulin or X10 (Novo Nordisk) were implanted s.c. in the diabetic mice. The minipumps released human insulin or X10 by constant s.c. infusion at doses of 0.6 or $0.9 \mathrm{nmol}_{\text {mouse }}^{-1}$ day $^{-1}$, which was approximately 27 and $41 \mathrm{nmol} \mathrm{kg}^{-1}$ day $^{-1}$, respectively. The non-diabetic mice were treated with vehicle via osmotic minipumps. On the same day, COLO-205 cells were injected s.c. in the right flank. Growth of the COLO-205 xenografts was monitored as described for experiment E. Blood glucose was measured for all mice on days 4,11 and 15, and was used to calculated the mean blood glucose for each mouse. At completion of the experiment, COLO-205 xenografts were collected from all mice, weighed and snap-frozen in liquid nitrogen for subsequent assessment of insulin receptor, IGF-1R and Akt activation, and epididymal fat pads were weighed. The design and analysis of the experiment are described in detail in ESM Methods and ESM Tables 4-5.

Preparation of tissue lysates All tissue and COLO-205 cell xenograft samples were lysed in Cell Extraction Buffer (Invitrogen, Carlsbad, CA, USA), supplemented with 4-(2aminoethyl)benzenesulfonyl fluoride hydrochloride and protease inhibitor cocktail (Sigma-Aldrich, Brøndby, Denmark) using a Qiagen Tissue Lyzer II (Qiagen, Copenhagen, Denmark). Protein concentration of the supernatant fraction was assessed with the Pierce bicinchoninic acid protein assay kit according to the manufacturer's instructions (Thermo Fisher Scientific, Hvidovre, Denmark).

Assessment of insulin receptor, IGF-1 receptor and Akt activation in liver, muscle, colon and COLO-205 xenografts The levels of insulin receptor, IGF-1R and Akt activation were assessed in tissue and xenograft samples from mice and rats using the commercially available ELISA kits listed in Table 2, according to the manufacturers' instructions. The specificity of the phospho-IGF-I receptor $\beta$ (Tyr1131) ELISA was validated as described in the ESM Methods. Additionally, levels of activated insulin receptor and IGF-1R were assessed in rat tissue samples by sandwich ELISAs as described in the ESM Methods with two novel capture antibodies: the monoclonal human anti-insulin receptor D2 antibody and the monoclonal rabbit/human chimeric anti-IGF-1R 226 antibody. Both antibodies were generated and characterised at Novo Nordisk (N. Blume et al, unpublished data).

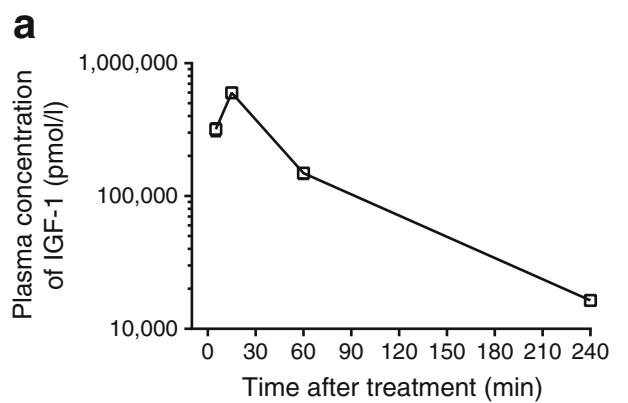

Fig. 2 Time course for IGF-1R activation in COLO-205 xenografts. (a) Treatment of nude mice with human IGF-1 (squares), $600 \mathrm{nmol} / \mathrm{kg}$ by s.c. injection, resulted in peak plasma exposure levels of $\sim 600,000 \mathrm{pmol} / \mathrm{l}$. The $y$-axis is on logarithmic scale $\left(\log _{10}\right)$. Symbols and bars are mean \pm SEM ( $n=8$ [IGF-1 at $5 \mathrm{~min}$ ] or $n=7$ [all other groups]). (b) Treatment with IGF-1, $600 \mathrm{nmol} / \mathrm{kg}$ (squares), decreased blood glucose compared with vehicle (circles). Symbols and bars are mean $\pm \operatorname{SEM}(n=8[$ IGF-1 at
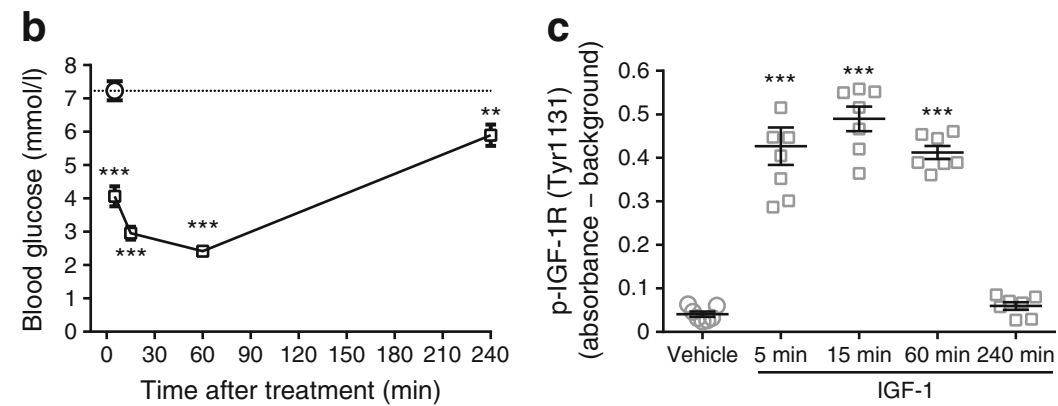

$5 \min$ ] or $n=7$ [all other groups]). (c) Significant activation of the IGF-1R was observed in COLO-205 xenografts in the same mice 5 min after treatment; by 240 min after treatment, IGF-1R activation had returned to the basal level. Symbols are observations from individual animals, horizontal lines are mean $\pm \operatorname{SEM}(n=8$ [IGF-1 at $5 \mathrm{~min}$ ] or $n=7$ [all other groups]). ${ }^{*} p<0.01$ and $* * * p<0.001$ vs the vehicle-treated group 


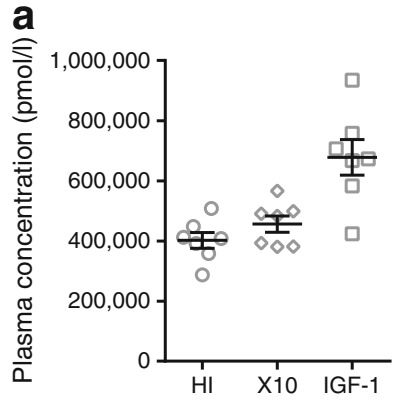

b

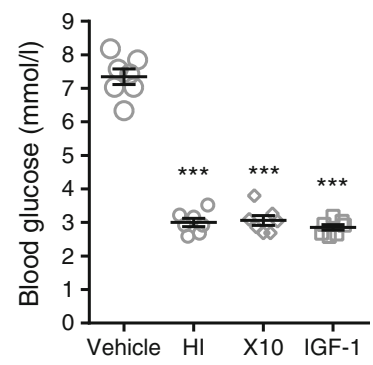

C

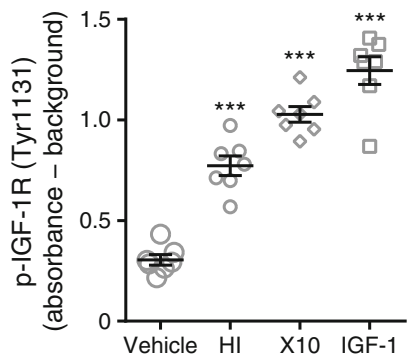

Fig. 3 Acute activation of IGF-1R by human insulin, X10 and IGF-1 in COLO-205 xenografts. (a) Treatment of nude mice with human insulin (circles), X10 (diamonds) or human IGF-1(squares) resulted in plasma exposure levels of 400,000 to $600,000 \mathrm{pmol} / 115 \mathrm{~min}$ after s.c. injection. (b) Treatment with human insulin, X10 and IGF-1 decreased blood glucose significantly compared with vehicle (large circles). (c) Treatment

Assay of blood glucose and plasma concentration of human insulin, X10, IGF-1 and mouse insulin Blood glucose and plasma concentrations of test compounds and endogenous mouse insulin were assessed as described previously [18].

Statistical analysis Statistical analysis of data was performed with the software GraphPad Prism (GraphPad Software, La with all three compounds increased levels of phospho-IGF-1R in the COLO-205 xenografts significantly compared with vehicle-treated mice. Symbols are observations from individual animals, horizontal lines are mean $\pm \operatorname{SEM}(n=7) . * * * p<0.001$ vs the vehicle-treated group. HI, human insulin

Jolla, CA, USA) or SAS JMP (SAS Institute, Cary, NC, USA). GraphPad Prism was used to fit dose-response curves describing thymidine incorporation in COLO-205 cells and calculate $\mathrm{EC}_{50}$ values.

Data from animal experiments A, B, C and D were analysed in GraphPad Prism, with one-way ANOVA for each endpoint, followed by pairwise comparison of each group a

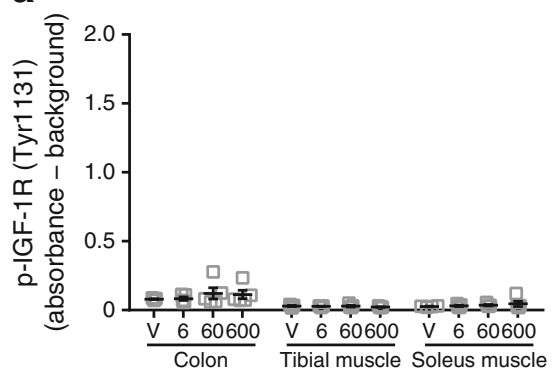

d

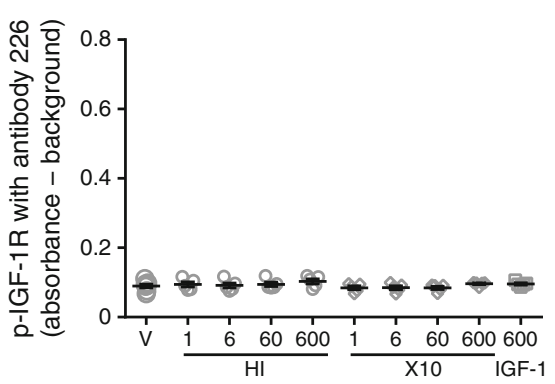

b

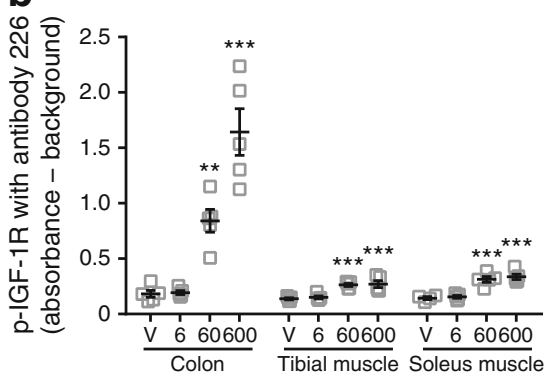

e

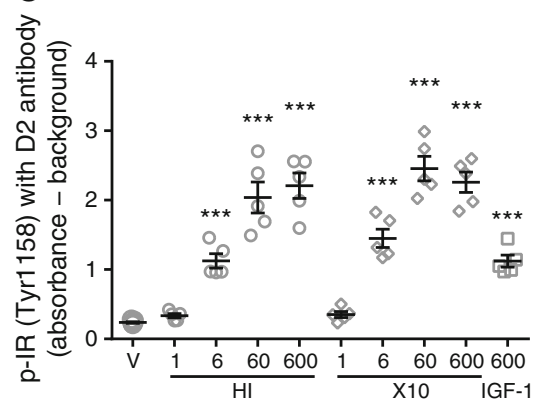

C
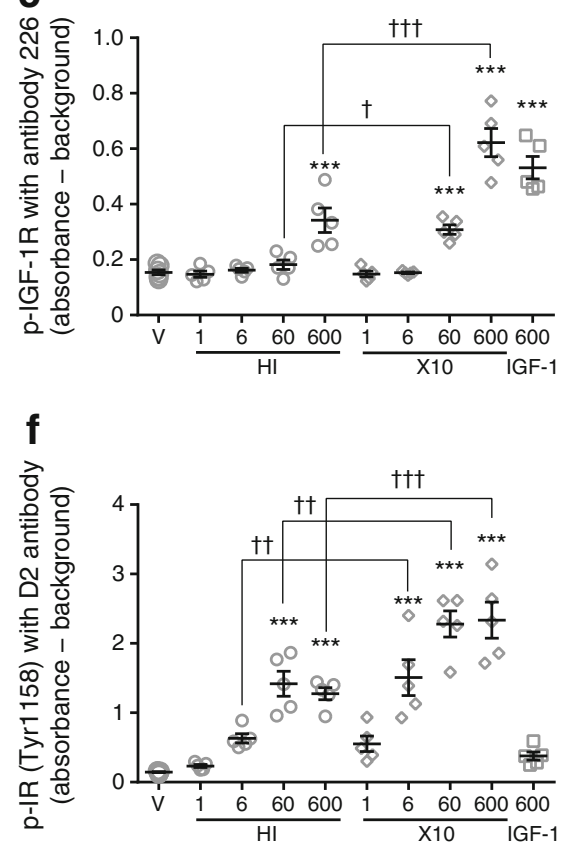

Fig. 4 IGF-1R activation in rat tissues. (a) In rats treated with human IGF-1 in doses of 6,60 or $600 \mathrm{nmol} / \mathrm{kg}$, no increased activation of the IGF-1R could be detected in samples of colon, anterior tibial muscle or the soleus muscle using the phospho-IGF-1R (Tyr1131) ELISA. (b) Compared with treatment with vehicle, treatment with human IGF-1 in doses of 60 and $600 \mathrm{nmol} / \mathrm{kg}$ increased IGF-1R activation in colon, the anterior tibial muscle and the soleus muscle when assessed with an IGF$1 \mathrm{R}$ capture antibody generated in-house. (c) Rats were treated with vehicle, human insulin or X10 in doses of 1, 6, 60 or $600 \mathrm{nmol} / \mathrm{kg}$, or IGF-1, $600 \mathrm{nmol} / \mathrm{kg}$, and treatment with human insulin or X10 resulted in significantly increased activation of IGF-1R in the colon. (d) Treatment with human insulin, X10 or IGF-1 did not result in IGF-1R activation in the liver. (e) Significantly increased activation of the insulin receptor was observed in the colon following treatment with human insulin, X10 or IGF-1. (f) Significantly increased activation of the insulin receptor was observed in the liver following treatment with human insulin, X10 or IGF-1. The $x$-axes show doses in $\mathrm{nmol} / \mathrm{kg}$. Symbols are observations from individual animals. Horizontal lines are mean \pm SEM $(n=7$ [vehicle] or $n=5$ [all other groups]). $* * p<0.01$ and $* * * p<0.001$ vs the vehicle-treated group. ${ }^{\dagger} p<0.05,{ }^{\dagger \dagger} p<0.01$ and ${ }^{\dagger \dagger} p<0.001$ as shown. HI, human insulin; IR, insulin receptor; V, vehicle 


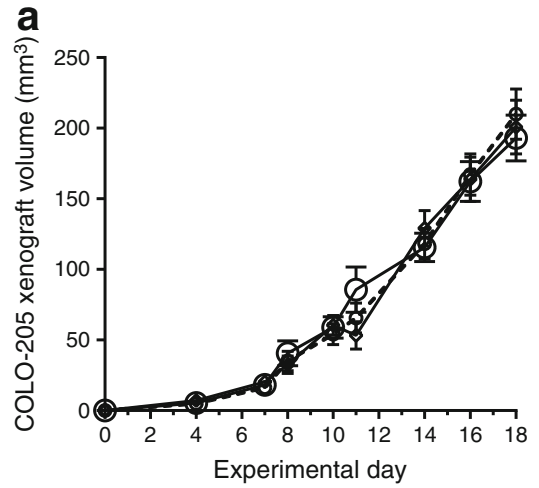

Fig. 5 Growth of COLO-205 xenografts in mice treated with human insulin or X10. (a) Mean volume \pm SEM of the COLO-205 xenografts during the experiment in mice treated with vehicle (large circles, $n=54$ ), human insulin $600 \mathrm{nmol} / \mathrm{kg}$ (small circles, $n=61$ ) or X10 $600 \mathrm{nmol} / \mathrm{kg}$

treated with a test compound with the vehicle-treated group, with Dunnett's correction for multiple comparisons. In experiment $\mathrm{D}$, groups treated with equimolar doses of human insulin and X10 were also compared and the Šidák correction for multiple comparisons was used.

Experiments $\mathrm{E}$ and $\mathrm{F}$ were analysed in SAS JMP. Each endpoint was analysed in a general linear model with treatment and block (experiment E) as explanatory variables. Differences between groups were expressed as a ratio (i.e. fold difference), and the analyses were therefore performed on logarithmically transformed data as described previously [19]. As adjustment for multiple pairwise comparisons of groups, Bonferonni (experiment E) or Tukey (experiment F) correction was used. In experiment $\mathrm{F}$, the correlation between xenograft mass and mean blood glucose was calculated with GraphPad Prism. A value of $p<0.05$ was considered statistical significant.

\section{Results}

Mitogenic potency of human insulin, X10 and IGF-1 in COLO205 cells in vitro The mitogenic potency of IGF-1 was found to be approximately 23 -fold higher than the mitogenic potency of human insulin in COLO-205 cells, while the mitogenic potency of X10 was approximately 6.5 -fold higher than human insulin (Fig. 1). This is in good agreement with previous findings that COLO-205 cells express six- to sevenfold higher levels of IGF-1R than insulin receptor (predominantly isoform A of insulin receptor) $[16,20]$.

Acute treatment with human insulin, $\mathrm{X} 10$ and IGF-1 results in activation of the IGF-1R in COLO-205 xenografts Treatment of mice bearing COLO-205 xenografts with human IGF-1, $600 \mathrm{nmol} / \mathrm{kg}$ by s.c. injection, resulted in peak plasma IGF-1 levels of approximately $600,000 \mathrm{pmol} / \mathrm{l}$ and significant lowering of blood glucose $(p<0.001)$ (Fig. 2a,b). With the b

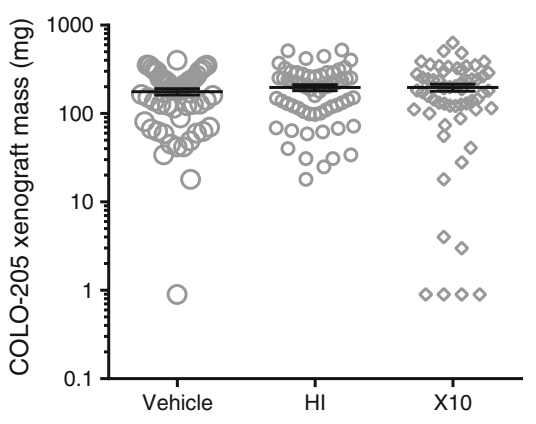

(diamonds, $n=59$ ). (b) Mass of the COLO-205 xenografts at completion of the experiment. The $y$-axis is on logarithmic scale $\left(\log _{10}\right)$. Symbols are observations from individual animals. Horizontal lines are mean \pm SEM (vehicle: $n=54$, human insulin: $n=61, \mathrm{X} 10: n=59$ ). HI, human insulin

specific phospho-IGF-1 receptor $\beta$ (Tyr1131) ELISA, which does not recognise phospho-sites on the insulin receptor (ESM Fig. 1), we demonstrated significant activation of IGF-1R in the COLO-205 xenografts at $5 \mathrm{~min}$ after treatment, with maximum activation after $15 \mathrm{~min}$ (Fig. 2c). Next, we explored the acute activation of IGF-1R in COLO-205 xenografts after treatment with $600 \mathrm{nmol} / \mathrm{kg}$ human insulin, X10 or IGF-1. All three compounds decreased blood glucose and resulted in plasma concentrations of approximately $500,000 \mathrm{pmol} / 1$ (Fig. 3a,b). Human insulin, X10 and IGF-1 all resulted in significant activation of IGF-1R in the COLO-205 xenografts 15 min after treatment $(p<0.001$, Fig. $3 c)$. In the gastrocnemius muscle and colon from the same animals, no detectable IGF-1R activation was observed with the phospho-IGF-1R (Tyr1131) ELISA (ESM Fig. 2). As IGF-1R is expressed in these tissues, this shows that the phospho-IGF-1R (Tyr1131) ELISA does not recognise phospho-sites on the rodent IGF$1 R$.

Activation of IGF-1R in rat colon and skeletal muscle IGF-1R activation in colon, the anterior tibial and soleus muscle from rats treated with IGF-1 was examined using the commercially

Table 3 Mean fold difference between groups for mass and volume of COLO-205 cell xenografts at euthanasia

\begin{tabular}{lll}
\hline Endpoint/comparison & Mean ratio & $95 \%$ CI \\
\hline Xenograft mass & & \\
HI vs vehicle & 1.07 & $0.74,1.54$ \\
X10 vs vehicle & 1.07 & $0.73,1.56$ \\
X10 vs HI & 1.00 & $0.70,1.45$ \\
Xenograft end volume & & \\
HI vs vehicle & 1.01 & $0.74,1.38$ \\
X10 vs vehicle & 1.18 & $0.85,1.63$ \\
X10 vs HI & 1.16 & $0.85,1.59$ \\
\hline
\end{tabular}

HI, human insulin 
a

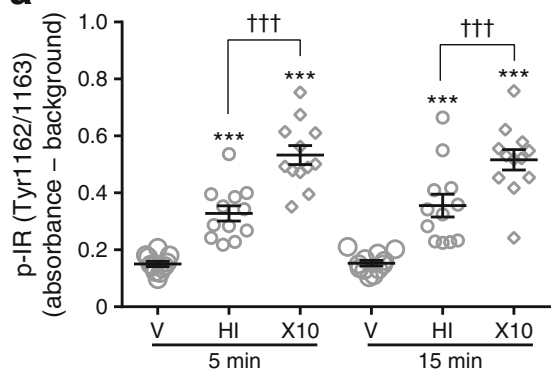

b

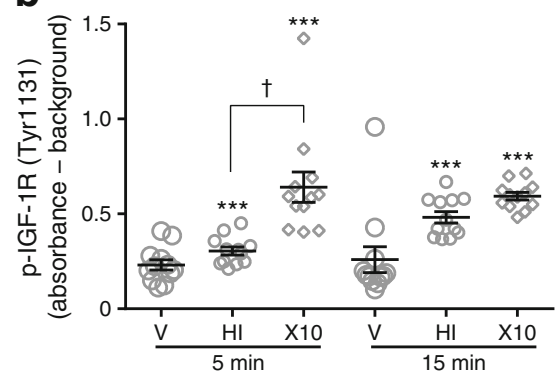

C

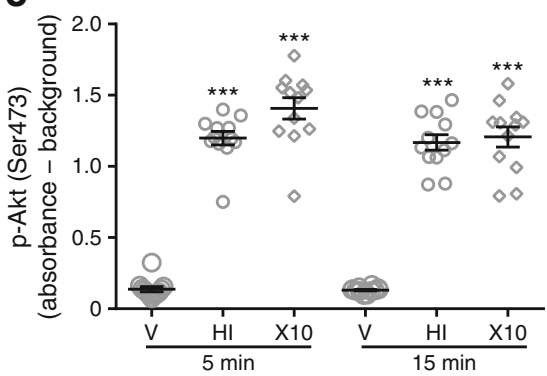

Fig. 6 Activation of insulin receptor, IGF-1R and Akt in COLO-205 xenografts in mice treated with vehicle (large circles), human insulin $600 \mathrm{nmol} / \mathrm{kg}$ (small circles) or X10 $600 \mathrm{nmol} / \mathrm{kg}$ (diamonds) for 18 days, assessed 5 or $15 \mathrm{~min}$ after the last treatment. (a) Activation of insulin receptor assessed with phospho-insulin receptor (Tyr1162/1163) ELISA. (b) Activation of IGF-1R assessed with phospho-IGF-1R (Tyr1131)
ELISA. (c) Activation of Akt, assessed with phospho-Akt (Ser473) ELISA. Symbols are observations from individual animals. Horizontal lines are mean $\pm \operatorname{SEM}(n=12) . * * * p<0.001$ vs the vehicle-treated group. ${ }^{\dagger} p<0.05$ and ${ }^{\dagger \dagger \dagger} p<0.001$ as shown. HI, human insulin; IR, insulin receptor; V, vehicle available phospho-IGF-1R (Tyr1131) ELISA and with a sandwich ELISA generated in-house, which could also capture rodent IGF-1R. No activation could be detected with the phospho-IGF-1R (Tyr1131) ELISA (Fig. 4a), whereas IGF$1 \mathrm{R}$ activation was observed in all three tissues with the inhouse sandwich ELISA (Fig. 4b). We next explored IGF-1R activation in rats treated with human insulin or X10 in doses of $1-600 \mathrm{nmol} / \mathrm{kg}$. Treatment with human insulin $600 \mathrm{nmol} / \mathrm{kg}$ and X10 60 and $600 \mathrm{nmol} / \mathrm{kg}$ resulted in IGF-1R activation in rat colon, with X10 a more potent activator than human insulin (Fig. 4c). In the liver, where the IGF-1R is expressed at very low levels, no IGF-1R activation could be detected (Fig. 4d). Pronounced activation of insulin receptor was observed after treatment with human insulin and X10 at doses of $6 \mathrm{nmol} / \mathrm{kg}$ and higher in both colon and liver (Fig. 4e,f). This, in combination with the absence of detectable IGF-1R activation in the liver, shows that the sandwich ELISA for activated IGF-1R with anti-IGF-1R antibody 226 does not recognise activated insulin receptor.

Treatment with human insulin and X10 does not influence growth of COLO-205 xenografts, despite activation of insulin and IGF-1 receptors Growth of the COLO-205 xenografts during experiment $\mathrm{E}$ and mass of the tumour xenografts at the end of the experiment are shown in Fig. 5a,b. Mean fold differences between the groups are shown in Table 3. The growth rates of all three groups were comparable and no significant differences were observed between any of the groups for xenograft volume or mass at the end of the experiment. Activation of insulin receptor, IGF-1R and Akt was assessed 5 or $15 \mathrm{~min}$ after the last treatment on experimental day 18 (Fig. 6 and Table 4). Treatment with both human insulin and X10
Table 4 Mean fold difference between groups for phospho-insulin receptor, phospho-IGF-1R and phospho-Akt in COLO-205 xenografts

\begin{tabular}{|c|c|c|c|c|}
\hline \multirow[t]{2}{*}{ Endpoint/comparison } & \multicolumn{2}{|c|}{$\begin{array}{l}\text { COLO- } 205 \text { xenograft } 5 \text { min after } \\
\text { treatment }\end{array}$} & \multicolumn{2}{|c|}{$\begin{array}{l}\text { COLO-205 xenograft } 15 \text { min after } \\
\text { treatment }\end{array}$} \\
\hline & Mean ratio & $95 \% \mathrm{CI}$ & Mean ratio & $95 \% \mathrm{CI}$ \\
\hline \multicolumn{5}{|c|}{ Phospho-insulin receptor (Tyr1162/1163) } \\
\hline HI vs vehicle & 2.2 & $1.7,2.8$ & 2.2 & $1.7,2.9$ \\
\hline X10 vs vehicle & 3.5 & $2.8,4.5$ & 3.5 & $2.5,4.4$ \\
\hline $\mathrm{X} 10$ vs HI & 1.6 & $1.3,2.1$ & 1.5 & $1.1,2.0$ \\
\hline \multicolumn{5}{|c|}{ Phospho-IGF-1R (Tyr1131) } \\
\hline HI vs vehicle & 1.4 & $1.0,1.9$ & 2.3 & $1.6,3.1$ \\
\hline $\mathrm{X} 10$ vs vehicle & 2.8 & $2.0,3.9$ & 2.8 & $2.0,3.9$ \\
\hline X10 vs HI & 2.0 & $1.5,2.8$ & 1.2 & $0.9,1.7$ \\
\hline \multicolumn{5}{|l|}{ Phospho-Akt (Ser473) } \\
\hline HI vs vehicle & 9.3 & $7.2,12.0$ & 8.9 & $7.4,10.7$ \\
\hline X10 vs vehicle & 10.8 & $8.4,14.0$ & 9.1 & $7.6,11.0$ \\
\hline X10 vs HI & 1.2 & $0.9,1.5$ & 1.0 & $0.9,1.2$ \\
\hline
\end{tabular}

HI, human insulin 


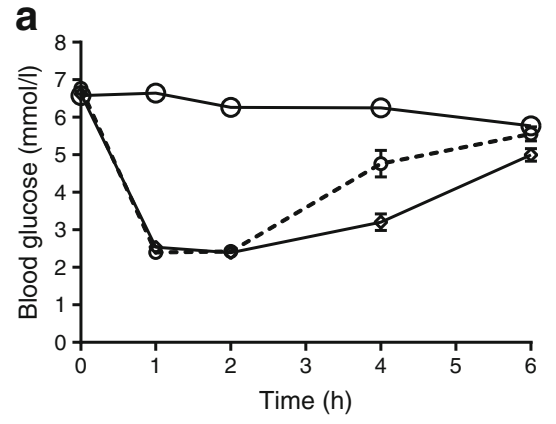

Fig. 7 Effect on blood glucose, body weight gain and epididymal fat mass after treatment with vehicle (large circles), human insulin $600 \mathrm{nmol} / \mathrm{kg}$ (small circles) or X10 $600 \mathrm{nmol} / \mathrm{kg}$ (diamonds) for 18 days. (a) Treatment with human insulin and X10 decreased blood glucose compared with vehicle, X10 more potently than human insulin, in agreement with the data describing the area above the blood glucose curve (Table 5). Symbols are mean $\pm \operatorname{SEM}(n=15$ [vehicle] or $n=30$ [human insulin and

resulted in significant acute activation of insulin receptor, IGF-1R and Akt in the COLO-205 xenografts after treatment over the previous 17 days.

\section{Treatment with X10 has stronger effect than human insulin on blood glucose, body weight gain and epididymal fat mass} The acute effect of treatment with human insulin and X10 on blood glucose, change in body weight during the experiment and mass of the epididymal fat pads at termination of experiment $\mathrm{E}$ is shown in Fig. 7; mean ratios between the groups are shown in Table 5. The area above the curve describing blood glucose was significantly increased in X10-treated animals by $\sim 20 \%$ ( $p=0.0058$ ). Consistent with this, the X10-treated animals gained significantly more body weight during the experiment than animals treated with human insulin $(p<0.001)$, and mass of the epididymal fat pads was significantly increased by $\sim 20 \%$ compared with animals treated with human insulin $(p=0.0016)$.

\section{Normalisation of blood glucose in diabetic mice with human insulin or X10 does not enhance growth of COLO-205 xeno-} grafts The effects of treatment of diabetic nude mice with human insulin or X10, administered for 15 days by continuous s.c. infusion, are shown in Fig. 8. Treatment with human insulin or X10 resulted in lowering of blood glucose from a hyperglycaemic to a normoglycaemic range (Fig. 8a), but did not significantly influence growth or mass of the COLO205 xenografts (Fig. 8b,c). There was significantly greater activation of the insulin receptor in mice treated with X10 than in the vehicle-treated normoglycaemic mice $(p<0.001)$ and the human-insulin-treated mice $(p=0.0039$ and $p<0.001$ at doses of 0.6 and 0.9 nmol mouse ${ }^{-1}$ day $^{-1}$, respectively, Fig. 8d), whereas no significant differences were found between the groups for activation of IGF-1R or Akt (ESM Fig. 3). Mass of the epididymal fat was significantly increased in mice treated with X10 0.9 nmol mouse ${ }^{-1}$ day $^{-1}$ compared with the
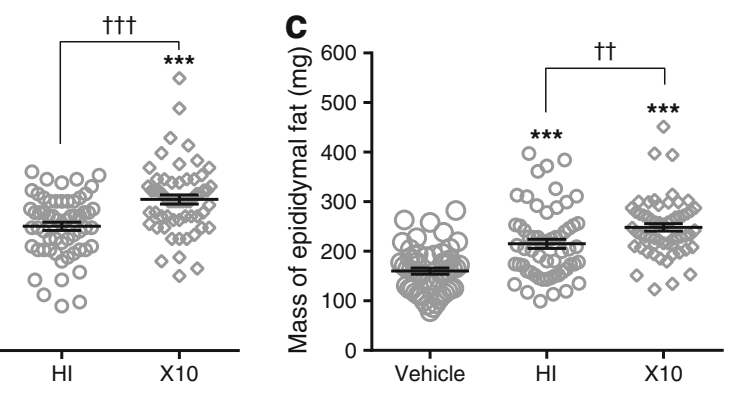

X10]). (b) X10 increased body weight in the mice more than treatment with human insulin and vehicle. (c) Human insulin and X10 increased mass of the epididymal fat depot in mice compared with vehicle, X10 more potently than human insulin. Symbols in (b) and (c) represent individual animals. Horizontal lines are mean $\pm \operatorname{SEM}(n=54$ [vehicle] or $n=$ 59 [human insulin and X10]). *** $p<0.001$ vs the vehicle-treated group. ${ }^{\dagger} p<0.01$ and ${ }^{\dagger \dagger} p<0.001$ as shown. HI, human insulin

vehicle-treated mice ( $p=0.0233$, Fig. 8e). Finally, for 27 mice with mean blood glucose in the range $2-5 \mathrm{mmol} / 1$ there was a significant negative correlation between xenograft mass and mean blood glucose $(p=0.0002$, Fig. 8 f, ESM Methods and ESM Table 5), i.e., hypoglycaemia did not inhibit growth of COLO-205 xenografts.

\section{Discussion}

The principal findings in our study are: (1) treatment with supra-pharmacological doses of human insulin and X10 can activate the IGF-1R in both human colon cancer xenografts and normal rodent tissues in vivo; (2) treatment of diabetic animals with pharmacologically relevant doses of human insulin and X10 does not activate the IGF-1R in COLO-205 xenografts; and (3) neither supra-pharmacological doses or

Table 5 Mean fold difference between groups for area above curves describing blood glucose, body weight and mass of epididymal fat

\begin{tabular}{lcc}
\hline Endpoint/comparison & Mean ratio & $95 \%$ CI \\
\hline Area above blood glucose curve & & \\
0-6 h after treatment (baseline: vehicle) & 1.21 & \\
X10 vs HI & $1.06,1.39$ \\
Body weight on experimental day 18 & \\
HI vs vehicle & 1.00 & $0.98,1.01$ \\
X10 vs vehicle & 1.02 & $1.01,1.04$ \\
X10 vs HI & 1.03 & $1.01,1.04$ \\
Epididymal fat mass on experimental day 18 & $1.17,1.52$ \\
HI vs vehicle & 1.33 & $1.39,1.80$ \\
X10 vs vehicle & 1.58 & $1.04,1.35$ \\
X10 vs HI & 1.19 & \\
\hline
\end{tabular}

HI, human insulin 
doses of human insulin or X10 with pharmacological relevance enhance growth of COLO-205 colon cancer xenografts.

Our findings are in clear contrast to recent studies, which reported that none of human insulin, X10 or a nonmetabolisable analogue of insulin glargine in suprapharmacological doses up to $1200 \mathrm{nmol} / \mathrm{kg}$ was able to activate the IGF-1R in normal rats and mouse allograft models [12-15]. However, there are several differences in methods between our study and the previous ones. First, the previous studies used either western blotting with an antibody that recognises phospho-epitopes on the IGF-1R as well as the insulin receptor (i.e. a non-specific antibody) or immunoprecipitation for detection of activated IGF-1R [12-15]. We used ELISAs, which are considered more suited for quantitative assessment than western blotting and immunoprecipitation. Furthermore, Gallagher et al $[12,13]$ do not state whether the IGF-1R activation observed in tumour allografts from IGF-1-treated mice represent the basal activation state after repeated treatment for 14 days, or activation seen shortly after an acute treatment. We demonstrate that activation of IGF-1R clearly depends on time. Tennagels et al [14] state that IGF-1R activation was assessed in rat skeletal muscle and mammary gland tissue 60 min after s.c. injection of human insulin or X10, but the positive control samples from IGF-1-treated rats were collected $5 \mathrm{~min}$ after treatment by i.v. injection. In that study, it is therefore possible that IGF-1R activation could have occurred in the human insulin- and X10-treated rats before the time for collection of tissue samples.

The clinical relevance of growth-stimulatory effects of insulin and insulin analogues is unknown, as they have only been observed in vitro and after treatment with suprapharmacological doses in animal models. However, a
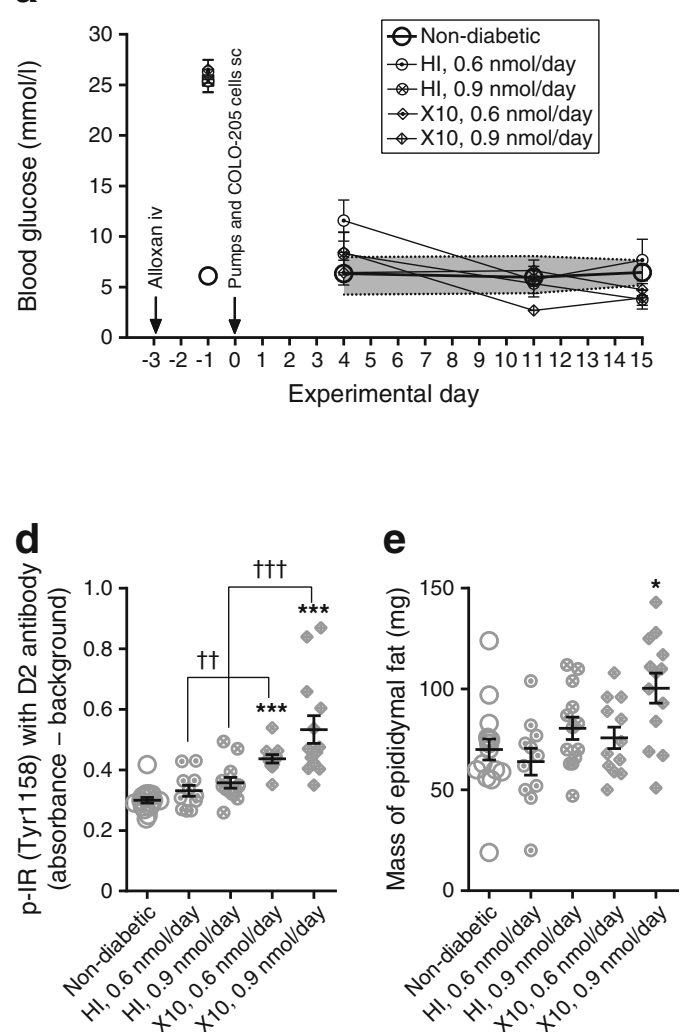

Fig. 8 Effect of normalisation of blood glucose with human insulin or X10 in diabetic nude mice with COLO-205 xenografts. (a) Treatment of diabetic nude mice with 0.6 or $0.9 \mathrm{nmol}$ mouse $^{-1}$ day $^{-1}$ of human insulin or X10 via osmotic minipumps resulted in normalisation of blood glucose. The shaded grey area shows the range of blood glucose in the normal non-diabetic nude mice. (b) Growth of COLO-205 xenografts was not influenced by treatment with human insulin or X10. (c) Mass of COLO-205 xenografts at completion of the experiment was not influenced by treatment with human insulin or X10. The $y$-axis is on logarithmic scale $\left(\log _{10}\right)$. (d) Treatment with X10 increased activation of insulin receptor significantly compared with non-diabetic mice and mice treated with an equimolar dose of human insulin. (e) Treatment with X10, $0.9 \mathrm{nmol} /$ day, increased mass of the epididymal fat significantly
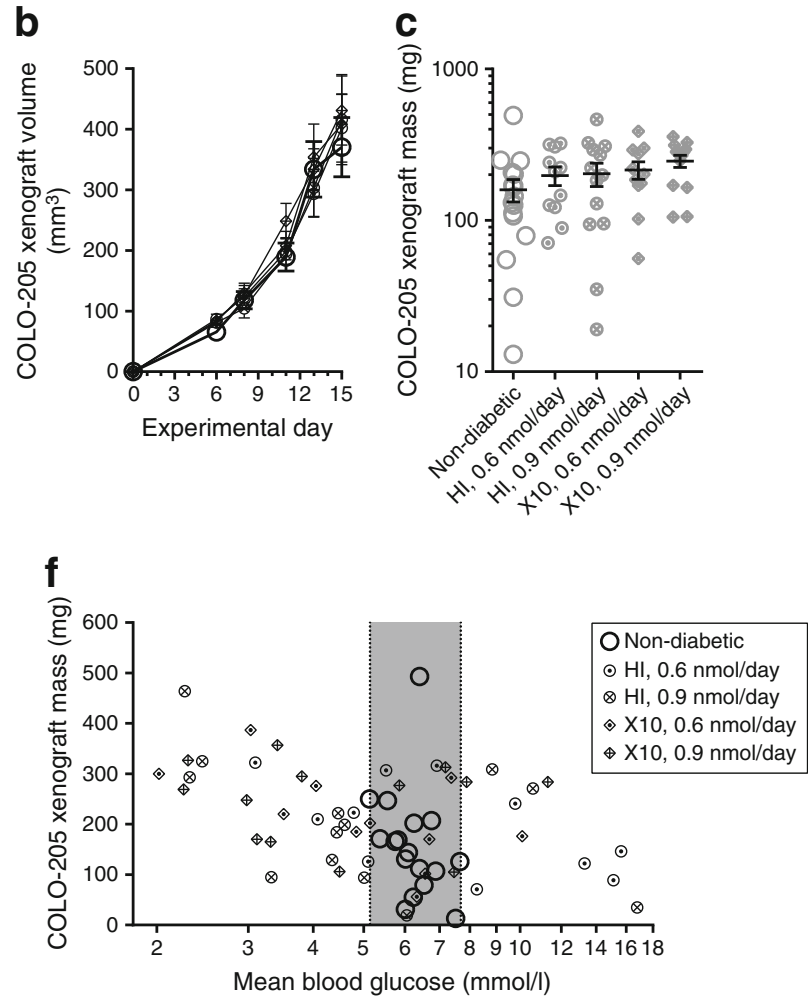

compared with non-diabetic mice. (f) In the hypoglycaemic range from 2 to $5 \mathrm{mmol} / \mathrm{l}$ there was a significant negative correlation between mass of COLO-205 xenograft and mean blood glucose. The $x$-axis is on logarithmic scale $\left(\log _{2}\right)$ to allow for detailed view in the hypoglycaemic range. The shaded grey area indicates the range of mean blood glucose in the non-diabetic mice. Symbols in (a) and (b) indicate mean values and error bars show the $\operatorname{SEM}(n=17$ [non-diabetic], $n=11$ [human insulin $0.6 \mathrm{nmol}$ mouse $^{-1}$ day $^{-1}$ ], $n=13$ [human insulin 0.9 nmol mouse $^{-1}$ day $^{-1}$ ], $n=11$ $\left[\mathrm{X} 10,0.6 \mathrm{nmol}\right.$ mouse $\left.{ }^{-1} \mathrm{day}^{-1}\right]$ and $n=13[\mathrm{X} 10,0.9 \mathrm{nmol}$ mouse $^{-1}$ day $\left.\left.^{-1}\right]\right)$. In (c-f), symbols indicate observations from individual animals; horizontal lines show the mean \pm SEM. $* p<0.05$ and $* * * p<$ 0.001 vs the non-diabetic group. ${ }^{\dagger} p<0.01$ and ${ }^{\dagger \dagger} p<0.001$ as shown. HI, human insulin; IR, insulin receptor 
development of X10 for therapeutic use was discontinued because of increased mitogenic potential in vitro and increased tumour incidence in vivo. The rationale behind this decision was that the interests of the patient should prevail if there is any doubt about the safety of a novel compound. Even though we demonstrate here that growth of COLO-205 xenografts was also not increased after treatment of diabetic mice with pharmacologically relevant doses (as hyperglycaemia was normalised), it is impossible to know if a mouse experiment of 15 days' duration reflects all aspects and risks related to treatment in a diabetic individual in a period of maybe 30 years or more. Our finding of no stimulation of growth of COLO-205 xenografts therefore does not mean that insulin and insulin analogues are never able to stimulate growth of cancer cells. However, it does indicate that insulin and insulin analogues are weak mitogenic compounds in vivo.

It is necessary to understand the mechanisms behind the increased mitogenic and growth-stimulatory effect of X10 in order to avoid developing novel insulin analogues with these characteristics. Possible mechanisms can be explored in vitro and in animal models, and as supra-pharmacological doses of $\mathrm{X} 10$ increased the mammary tumour incidence in female rats, it is relevant to explore how such high doses can stimulate growth of cancer cells. In our study, a supra-pharmacological dose of human insulin and X10 activated the IGF-1R and insulin receptor in COLO-205 xenografts, but growth of the xenografts was not affected by repeated treatment with this high dose for 18 days. In the COL0-205 xenograft model, it is therefore not possible to conclude whether increased IGF-1R activation by an insulin analogue correlates with increased growth or not. However, as we also demonstrate that X10 increases IGF-1R activation in rat tissues, it cannot be excluded that increased IGF-1R activation could explain why X10 increased the incidence of mammary tumours in female rats.

Our study also shows that the increased mitogenic potency of X10 in COLO-205 cells is an in vitro phenomenon that does not occur under in vivo conditions, neither after repeated treatment with supra-pharmacological doses nor after continuous treatment with pharmacological relevant doses in diabetic animals. Growth of COLO-205 cells in vivo must be stimulated by some factor(s) in serum other than exogenous human insulin and X10. This is supported by our finding from prolonged culture and repeated treatment of COLO-205 cells in vitro; with increasing concentrations of serum, the effect of adding human insulin or X10 disappeared (ESM Fig. 4).

One of the xenograft experiments in this study was performed in normoglycaemic host animals, in which treatment with high doses of insulin or insulin analogues induces hypoglycaemia. A potential concern could be that growthstimulatory effects of insulin and X10 are masked by the hypoglycaemia. However, the original finding with X10 was in a 12 month chronic toxicity study performed in normal rats, which developed hypoglycaemia with each X10 treatment, and in several previous allograft experiments in normal mice, treatment with high doses of insulin and X10 increased tumour growth despite repeated reduction of blood glucose to a hypoglycaemic level, fully comparable with this study [12, $13,18]$. Furthermore, in the COLO-205 xenograft experiment performed in diabetic host animals, we explored the correlation between xenograft mass and the mean blood glucose during the experiment days and observed the largest tumours in the mice with the lowest blood glucose and a significant negative correlation between tumor mass and blood glucose, i.e. hypoglycaemia did not inhibit growth of COLO-205 xenografts.

It was previously reported that IGF-1 increases proliferation of vascular smooth muscle cells and endothelial cells only under hyperglycaemic conditions [21], and it can be hypothesised that human insulin and X10 would enhance growth of COLO-205 xenografts under hyperglycaemic conditions. This could be relevant to explore, but as described above, this study focused on effects during normo- and hypoglycaemia. Furthermore, some animals treated with human insulin or X10 in experiment $\mathrm{F}$ had blood glucose ranging from $\sim 8$ to $17 \mathrm{mmol} / \mathrm{l}$, but the xenografts in these animals were not larger than xenografts in the normo- or hypoglycaemic animals (Fig. 8f), and in vitro human insulin and X10 have similar mitogenic potency in COLO-205 cells at glucose concentrations of 5 and $25 \mathrm{mmol} / \mathrm{l}$ (ESM Fig. 5). This could indicate that human insulin and X10 are not more mitogenic for COLO-205 xenografts during hyperglycaemia.

It could be speculated whether the single amino acid substitution in X10 compared with human insulin has any implication at all for the effects observed in vivo. However, it was possible to detect significant differences between the human insulin- and X10-treated groups in our study. Treatment with X10 resulted in significantly more activation of the insulin receptor and IGF-1R in liver, colon and COLO-205 xenografts than treatment with an equimolar dose of human insulin, in agreement with the higher binding affinity of X10 to the insulin receptor and IGF-1R [9]. The stronger activation of insulin receptors and IGF-1Rs correlated with other biological effects in the mice. The acute effect on blood glucose was increased by $\sim 20 \%$ in the X10-treated mice, and in good agreement with this, these mice gained more body weight than the mice treated with human insulin. The mass of the epididymal fat depot was also significantly increased by $\sim 20 \%$ in the X10-treated mice, and a comparable trend was observed in diabetic mice treated with the highest doses of human insulin and X10. Thus, it could be speculated that the increased mammary tumour incidence observed in the toxicology study with X10 [1] may have been an indirect effect of the increased body weight in these rats treated with high doses of X10, as obesity is associated with an increased risk for developing cancer [22]. 
Clarification of the mechanisms by which human insulin and insulin analogues can exert a growth-stimulating effect is important for the design of novel insulin analogues. We show here that IGF-1R activation does occur in vivo after treatment with a supra-pharmacological dose of human insulin and X10, but further studies are necessary to clarify if this characteristic is responsible for the increased tumour incidence observed with supra-pharmacological doses of X10. Preferably, such studies should be performed in model systems where a clear difference between human and X10 exists, if such in vivo models can be identified.

Acknowledgements We thank A. Bowman, S. L. Riisberg and K. S. Tornqvist for technical assistance with the animal experiments, and $\mathrm{P}$. Jensen, M. Stendal and C. H. Dahl for performing ELISAs for insulin receptor, IGF-1R and Akt activation (all at Insulin Research, Novo Nordisk, Maaloev, Denmark).

Data availability The data described in this manuscript are available, on request, from the corresponding author.

Funding This study was funded entirely by Novo Nordisk. Representatives from Novo Nordisk other than the authors provided editorial assistance only.

Duality of interest All authors are employees at Novo Nordisk and own shares in the company.

Contribution statement This study was conceived and designed by $\mathrm{HH}$, MSJ, NB, RS, AL, BFH. Acquisition of data was performed by HH, MSJ, $\mathrm{NB}, \mathrm{AL}$ and BFH. Data were analysed and interpreted by HH, MSJ, NB, $\mathrm{RS}, \mathrm{AL}$ and $\mathrm{BFH}$. The manuscript was drafted and revised by HH, MSJ, $\mathrm{NB}, \mathrm{RS}, \mathrm{AL}$ and BFH. All authors gave final approval of the version to be published. $\mathrm{HH}$ is the guarantor of this work.

\section{References}

1. Drejer K (1992) The bioactivity of insulin analogues from in vitro receptor binding to in vivo glucose uptake. Diabetes Metab Rev 8: 259-285

2. Hemkens LG, Grouven U, Bender R et al (2009) Risk of malignancies in patients with diabetes treated with human insulin or insulin analogues: a cohort study. Diabetologia 52:1732-1744

3. Jonasson JM, Ljung R, Talback M, Haglund B, Gudbjornsdottir S, Steineck G (2009) Insulin glargine use and short-term incidence of malignancies-a population-based follow-up study in Sweden. Diabetologia 52:1745-1754

4. Investigators OT, Gerstein HC, Bosch J et al (2012) Basal insulin and cardiovascular and other outcomes in dysglycemia. N Engl $\mathrm{J}$ Med 367:319-328

5. Adami HO, McLaughlin J, Ekbom A et al (1991) Cancer risk in patients with diabetes mellitus. Cancer Causes Control 2:307-314
6. Coughlin SS, Calle EE, Teras LR, Petrelli J, Thun MJ (2004) Diabetes mellitus as a predictor of cancer mortality in a large cohort of US adults. Am J Epidemiol 159:1160-1167

7. Gallagher EJ, LeRoith D (2015) Obesity and diabetes: the increased risk of cancer and cancer-related mortality. Physiol Rev 95:727748

8. Hansen BF, Danielsen GM, Drejer K et al (1996) Sustained signalling from the insulin receptor after stimulation with insulin analogues exhibiting increased mitogenic potency. Biochem J 315: 271-279

9. Kurtzhals P, Schaffer L, Sorensen A et al (2000) Correlations of receptor binding and metabolic and mitogenic potencies of insulin analogs designed for clinical use. Diabetes 49:999-1005

10. Slieker LJ, Brooke GS, DiMarchi RD et al (1997) Modifications in the B10 and B26-30 regions of the B chain of human insulin alter affinity for the human IGF-I receptor more than for the insulin receptor. Diabetologia 40(Suppl 2):S54-S61

11. Hansen BF, Glendorf T, Hegelund AC et al (2012) Molecular characterisation of long-acting insulin analogues in comparison with human insulin, IGF-1 and insulin X10. PLoS One 7:e34274

12. Gallagher EJ, Alikhani N, Tobin-Hess A et al (2013) Insulin receptor phosphorylation by endogenous insulin or the insulin analog AspB10 promotes mammary tumor growth independent of the IGF-I receptor. Diabetes 62:3553-3560

13. Gallagher EJ, Zelenko Z, Tobin-Hess A, Werner U, Tennagels N, LeRoith D (2016) Non-metabolisable insulin glargine does not promote breast cancer growth in a mouse model of type 2 diabetes. Diabetologia 59:2018-2025

14. Tennagels N, Welte S, Hofmann M, Brenk P, Schmidt R, Werner U (2013) Differences in metabolic and mitogenic signallingof insulin glargine and AspB10 human insulin in rats [corrected]. Diabetologia 56:1826-1834

15. Werner U, Korn M, Schmidt R, Wendrich TM, Tennagels N (2014) Metabolic effect and receptor signalling profile of a nonmetabolisable insulin glargine analogue. Arch Physiol Biochem 120:158-165

16. Lundby A, Bolvig P, Hegelund AC et al (2015) Surface-expressed insulin receptors as well as IGF-I receptors both contribute to the mitogenic effects of human insulin and its analogues. J Appl Toxicol 35:842-850

17. Committee for the Update of the Guide for the Care and Use of Laboratory Animals (2011) Guide for the Care and Use of Laboratory Animals. The National Academies Press, Washington DC

18. Hvid H, Blouin MJ, Birman E et al (2013) Treatment with insulin analog X10 and I GF-1 increases growth of colon cancer allografts. PLoS One 8:e79710

19. Keene ON (1995) The log transformation is special. Stat Med 14: $811-819$

20. Baricevic I, Jones DR, Roberts DL et al (2015) A framework for the in vitro evaluation of cancer-relevant molecular characteristics and mitogenic potency of insulin analogues. Carcinogenesis 36:1040 1050

21. Clemmons D, Maile L, Xi G, Shen X, Radhakrishnan Y (2011) IgfI signaling in response to hyperglycemia and the development of diabetic complications. Curr Diabetes Rev 7:235-245

22. Bianchini F, Kaaks R, Vainio H (2002) Overweight, obesity, and cancer risk. Lancet Oncol 3:565-574 